\title{
Associations Among Violence Victimization and/or Exploitation of Minors and Later Young Adulthood Violence
}

\author{
Hannabeth Franchino-Olsen ${ }^{1,2}$ (1) . Sandra L. Martin ${ }^{1} \cdot$ Carolyn T. Halpern $^{1} \cdot$ John S. Preisser $^{3} \cdot$ Catherine Zimmer $^{4}$. \\ Meghan Shanahan ${ }^{1}$
}

Accepted: 17 December 2021 / Published online: 19 January 2022

(c) The Author(s) 2022

\begin{abstract}
Little is known about how minor sex exchange (MSE) may impact violence victimization during adulthood. This study investigates potential associations between MSE and forms of interpersonal violence victimization in young adulthood, while controlling for additional forms of violence experienced in childhood and adolescence. Data from The National Longitudinal Study of Adolescent to Adult Health $(n=11,500)$ were used in this analysis. Multivariable logistic regression estimated associations between adolescent victimizations and interpersonal violence in young adulthood, while controlling for demographic variables, adolescent risk behaviors, and child maltreatment. Although multivariable models found that MSE was not significantly associated with either form of interpersonal violence in young adulthood, adolescent IPV and community violence significantly increased the likelihood of IPV in young adulthood, and adolescent community violence significantly increased the likelihood of community violence in young adulthood. Adolescent risk behaviors, child maltreatment, and certain demographic characteristics were also associated with violence in young adulthood. Prevention, intervention, and screening efforts should consider how violence victimization across the life course-from childhood to young adulthoodimpacts the experiences and needs of violence survivors.
\end{abstract}

Keywords Sex trafficking $\cdot$ Sex exchange $\cdot$ Minors $\cdot$ Violence $\cdot$ Victimization $\cdot$ Young adult

\section{Introduction}

In the United States, a minor (an individual under age 18) is considered to have experienced sex trafficking if they sell, trade, or exchange sex in a commercial sex act (Kotrla, 2010). Domestic minor sex trafficking (DMST) reflects any involvement of a minor (who is a citizen or legal permanent resident in the United States) in commercial sex, regardless of whether

Hannabeth Franchino-Olsen

hb.franchino-olsen@ed.ac.uk; hbo@unc.edu

1 Department of Maternal and Child Health, Gillings School of Global Public Health, University of North CarolinaChapel Hill, Chapel Hill, NC, USA

2 Social Work, School of Social and Political Science, University of Edinburgh, Edinburgh, Scotland, UK

3 Departmet of Biostatistics, Gillings School of Global Public Health, University of North Carolina-Chapel Hill, Chapel Hill, NC, USA

4 Odum Institute for Research in Social Science, University of North Carolina-Chapel Hill, Chapel Hill, NC, USA or not a third-party trafficker is involved, as minors cannot consent to engage in sex work (Kotrla, 2010; Trafficking Victims Protection Act, 2017; Trafficking Victims Protection Reauthorization Act, 2003; Trafficking Victims Protection Reauthorization Act, 2006; Trafficking Victims Protection Reauthorization Act, 2013; Victims of Trafficking \& Violence Protection Act, 2000; William Wilberforce Trafficking Victims Protection Reauthorization Act, 2008).

Substantial diversity exists in the nature and circumstances around a minor's involvement in DMST. Such engagement in commercial sex may include exchanging sex acts for money, drugs, food, housing/shelter, gifts, or other items of commercial value (Institute of Medicine (IOM) \& National Research Council (NRC), 2013). DMST may occur when minors are forced or coerced to engage in commercial sex by a third-party such as a romantic partner, family member, friend, acquaintance, or stranger (IOM \& NRC, 2013). DMST may also occur when minors choose to engage in commercial sex in order to meet basic needs or because other economic options (e.g., an hourly job) are unavailable or unappealing to them (IOM \& NRC, 2013; 
Showden \& Majic, 2018). In addition, minors may engage in commercial sex in order to support a substance dependency (Goldberg \& Moore, 2018; Showden \& Majic, 2018). All of these circumstances and currencies for minors' involvement in commercial sex qualify as DMST and reflect exploitation and abuse of vulnerable children and adolescents (Victims of Trafficking \& Violence Protection Act, 2000). Minor sex exchange (MSE), in which a minor exchanges or trades sex for something of commercial value, can be considered a type of DMST or descriptor of a DMST event and will be used in this study to consider minors who trade sex in the United States (O'Brien, 2017; Showden \& Majic, 2018).

While DMST and, more specifically, MSE occur across all fifty states and are not isolated to a particular gender or demographic group, no credible estimates exist for the overall number of DMST victims/survivors or the prevalence of DMST (Franchino-Olsen et al., 2020; Stransky \& Finkelhor, 2008). Given this, recent scholarship has recommended that researchers avoid citing faulty DMST estimates and that the anti-human trafficking field should leverage emerging methodologies to generate improved and more applicable estimates of trafficking in the United States and across the world (Franchino-Olsen et al., 2020; National Academies of Sciences Engineering \& Medicine, 2020).

Although DMST and MSE are forms of exploitation and abuse with potentially long-term consequences on survivors' physical and mental health (Le et al., 2018), we have a more limited understanding of how these experiences during childhood and adolescence may be linked to non-physical or mental health consequences and outcomes in adulthood, such as the impact of DMST on relationships, violence victimizations, education, and parenting. A review of the health issues associated with DMST found that substance use/abuse, mental health outcomes (including depression, post-traumatic stress disorder, suicidal behavior, psychiatric hospitalization, and trauma history), sexual and reproductive health outcomes (such as sexually transmitted infections, $\mathrm{HIV}$, and maternal or infant complications with pregnancy or birth outcomes), and physical health outcomes (injury, pain) were often experienced by individuals with a history of DMST (Le et al., 2018). Most previous studies were somewhat limited by the sampling and study designs which primarily relied on small convenience samples or other nonrepresentative samples and were typically cross-sectional or case-file review, rather than longitudinal, studies (Le et al., 2018). For example, Edinburgh et al. (2015) sampled 62 adolescents (aged 12-19) seen at an urban hospital-based child advocacy center who had a history of DMST or sexual exploitation. Walls and Bell (2011) sampled 1,625 minors and young adults (aged 10-25) who were homeless, in shelters, or on the street; of their sample, $9.4 \%$ reported experiencing MSE in order to meet basic needs. Reid and Piquero (2014) conducted a longitudinal study of 114 adolescents (aged 14-19) who reported MSE via ever being paid for sex. The limitations of these samples mean that much of the evidence for the correlates for and outcomes of DMST or MSE are not from large, representative and/or longitudinal samples.

In addition to these smaller scale studies (Le et al., 2018), data from the nationally representative National Longitudinal Study of Adolescent to Adult Health (Add Health) also have been analyzed to examine links between MSE in adolescence and outcomes during adulthood (Edwards et al., 2006; Kaestle, 2012; Ulloa et al., 2016). These Add Health studies focused on the adolescent health outcomes for those who had experienced MSE or on the health outcomes for minors and young adults engaged in commercial sex (i.e., MSE for minors, sex work for young adults) using a large, population-based sample. Similar to the results from small, non-representative studies, the studies examining Add Health data found adolescent health outcomes associated with MSE include sexually transmitted infections, poor mental health (including low self-worth and suicide ideation), and substance use/abuse (Edwards et al., 2006; Kaestle, 2012; Ulloa et al., 2016). In studies using longitudinal data, such as Add Health, there has been limited investigation into non-physical or mental health outcomes beyond a few years past age 18 or into early adulthood, so less is understood about some of the long-term outcomes of DMST or MSE (Edwards et al., 2006; Kaestle, 2012; Le et al., 2018; Ulloa et al., 2016). Additionally, no work has examined the impact of DMST or MSE on adulthood violence victimizations using a population-based sample.

The life course perspective may be helpful to understand potential interconnections between DMST or MSE experiences and subsequent outcomes in adulthood. The life course perspective recognizes some events as significant turning points that may redirect an individual's life path and impact subsequent life experiences (Elder, 1985). Because the life course perspective focuses on changes over time and considers how the experiences of childhood and/or adolescence may influence the events and health of young adulthood to old age, it has been applied to work examining violence revictimization or polyvictimization (Hendricks, 2012; Herrenkohl et al., 2020; Hing et al., 2021). Previous studies have demonstrated links between abuse and violence in childhood or adolescence and violence victimizations in adulthood (Costa et al., 2015; Fargo, 2009). Experiences of child abuse are associated with violence victimizations in adolescence, including intimate partner violence (IPV) or dating violence (Classen et al., 2005; Fargo, 2009). Violence victimizations during adolescence are, in turn, associated with experiencing violence in adulthood (Classen et al., 2005; Cui et al., 2013; Exner-Cortens et al., 2017; Fargo, 2009; Jouriles et al., 2017). These recurring violence victimizations-which may entail repeated instances of one type of 
abuse (e.g., sexual abuse/assault revictimization; Classen et al., 2005; Fargo, 2009) or involve multiple shifting forms of violence or polyvictimization (e.g., child sexual abuse to adolescent sexual assault to physical IPV; Classen et al., 2005; Costa et al., 2015) — can be viewed as connected via the life course perspective. Using the lens of the life course perspective, violence revictimization studies have considered how the experiences of violence in early life may impact subsequent violence, though previous studies have not yet considered whether experiences of DMST or MSE may follow similar patterns of revictimization across the life course (Fargo, 2009; Herrenkohl et al., 2020; Hing et al., 2021). Instances of DMST, including MSE, may serve as significant life events that impact an individual far beyond childhood and adolescence. Impacts stemming from DMST may include many types of adult health outcomes, including adulthood violence victimization (Le et al., 2018).

\section{Current study}

This study investigated violence victimization consequences during young adulthood (age 18-34) for those who experienced for MSE, with MSE capturing the exchange of sex for money or drugs. This investigation sought to expand the understanding of the outcomes of MSE beyond the commonly cited physical and mental health effects of minors involved in commercial sex. It examined whether experiencing MSE increased the likelihood of experiencing interpersonal violence victimization in young adulthood, including intimate partner violence and violence perpetrated by others (community violence) (Decker et al., 2018). IPV was defined as physical, psychological, or sexual harm perpetrated by a current or former partner (Decker et al., 2018). Community violence refers to physical fighting or assault with or without the use of firearms or weapons and typically occurs between strangers, friends, acquaintances, often outside of residential dwellings (Decker et al., 2018). Interpersonal violence captured any form of IPV and/or community violence victimization(s). In seeking to determine whether MSE had a long-term impact on relationships and experiences of violence long after MSE had occurred, this study sought connect MSE with violence later in the life course which could inform policy and practice efforts seeking to prevent and respond to minors who experience exploitation via commercial sex.

This study benefited from the use of Add Health data, which provided a large, population-based sample (originally nationally representative of adolescents) to assess the sensitive topics of violence victimization and the relatively rare occurrences of MSE. The design of Add Health provided generalizable findings and, based on previous publications using the data to investigate MSE (Edwards et al., 2006; Kaestle, 2012; Ulloa et al., 2016), likely detected individuals who experienced MSE or other forms of violence as school-enrolled adolescents who may have been overlooked in studies focused on minors considered more at risk for these victimizations (e.g., studies drawing samples from homeless or runaway minors). The diversity of the MSE sample was also enhanced by the relatively large sample of males in Add Health who indicated they had exchanged sex for money or drugs (Edwards et al., 2006; Kaestle, 2012; Ulloa et al., 2016). As males are frequently overlooked or understudied as having experiences of MSE, this relatively large sample of respondents with a history of MSE who are male added diversity to this sample (Robert \& Willis, 2013).

In particular, the study sought to answer the following questions:

1) Are MSE experiences associated with young adulthood interpersonal violence victimizations, including community violence and IPV?

Hypothesis 1: MSE experiences will be associated with increased odds of young adulthood interpersonal violence (community violence and/or IPV).

2) Beyond MSE, are additional forms of violence/abuse in childhood and adolescence associated with young adulthood interpersonal violence victimizations?

Hypothesis 2: Childhood and adolescent experiences of abuse and violence - excluding MSE-will be associated with increased odds of young adulthood interpersonal violence (community violence and/or IPV).

\section{Methods}

\section{Sample}

This study analyzed data from Add Health, a large, nationally representative, longitudinal study with a sample of more than 20,000 adolescents in the United States who were in 7th-12th grade in 1994-1995 (Harris et al., 2009).

The analysis data set included information from the first four waves of Add Health data collection, more specifically, information from Waves I and II when the respondents were adolescents, and from Waves III and IV when the respondents were young adults. Wave I included interview data with the original 20,743 respondents (10,480 females and 10,263 males) who were aged 12-20 years in 1994-1995; respondents were re-contacted in 1996 for the Wave II interviews, which included 13,568 respondents $(6,963$ females and 6,605 males) who were aged 12-22 years (Harris, 2013). All Wave I respondents were eligible to be included at Wave III interviews in 2001-2002, and 14,322 individuals $(6,759$ males and 7,563 females) aged 18-28 years were included with non-zero sampling weights. The Wave IV interview 
was conducted in 2008-2009 when participants were 24-34 years old and included 12,288 individuals $(5,604$ males and 6,684 females) who were also interviewed at Wave I and Wave III.

To be eligible for inclusion in the analysis data set, interview data for each respondent must have been collected during Waves I, III, and IV, and include non-zero longitudinal sampling weights. ${ }^{1}$ In addition, the respondents must have been 18 years old or younger at Wave I (if they were not included in Wave II) or at Wave II, and have information available on the demographic variables of race/ethnicity, adolescent family structure, and age at Wave IV.

\section{Measures}

Information about the Add Health measures used can be found in Supplemental Table A. MSE events were detected by asking respondents about their experiences of "giv[ing] someone sex in exchange for drugs or money." As young adults, they were asked about their recent experiences of interpersonal violence victimizations (community violence; IPV). Child maltreatment (physical abuse, physical neglect, or sexual abuse before $6^{\text {th }}$ grade) was retrospectively asked about in young adulthood. Adolescent violence victimizations, adolescent risk behaviors, and demographics were also included. For more information on adolescent variablesincluding violence, risk behaviors, and MSE-and demographic variables, please refer to (Franchino-Olsen et al., 2021).

\section{Analyses}

Descriptive statistics for the key variables of interest were conducted for the analysis sample. Bivariate logistic regressions examined the relationships between all predictors, including MSE, and young adulthood violence outcomes separately.

Multivariable logistic regression models investigated associations between MSE experiences and young adulthood violence outcomes, while controlling for adolescent, childhood, and demographic variables. Young adulthood violence outcomes for the models included any community violence (yes or no), any IPV (yes or no), and any interpersonal violence (yes or no). These violence outcomes were modeled as a function of adolescent variables, including MSE, adolescent violence victimizations (adolescent community violence, adolescent IPV), and adolescent risk behaviors (alcohol or drug use, shoplifting, running away

\footnotetext{
${ }^{1}$ Individuals who were high school seniors $\left(12^{\text {th }}\right.$ grade) in Wave I were not sampled at Wave II unless they were part of the genetic sample. However, they were returned to the sample for Waves III and IV.
}

from home), as well as demographic variables (biological sex, race/ethnicity, age, parental education, family structure).

Multiple imputation by chained equations (MICE) was conducted to account for item nonresponse on model variables in the eligible sample. Data underwent 100 imputations $(\mathrm{m}=100)$ using demographic variables (biological sex, race/ ethnicity, age, family structure) as predictors. All analyses account for the complex survey design of Add Healthincluding weighting, stratification, and clustering-and the use of MICE $(m=100)$ in the application of sampling weights (post-stratified grand sample longitudinal weight for Waves I, III, and $\mathrm{IV}^{2}$ ) and adjustment of variance estimates (Harris, 2013). Analyses were completed using Stata 16.1 (StataCorp, 2020).

\section{Results}

The eligible Add Health sample size included 11,500 respondents. Of the eligible respondents, approximately half had no missing data for all analysis variables ( $n_{\text {complete case }}=5,886$ ). The other half of the sample were eligible for inclusion but had missing responses for at least one variable of interest $\left(n_{\text {partially complete }}=5614\right)$. The following variables were missing responses for less than $2 \%$ of respondents: Minor sex exchange; Shoplifting; Running away from home; Highest parental education; Community violence at Wave III; Physical IPV at Wave IV; Psychological IPV at Wave IV; and Sexual IPV at Wave IV. The remaining variables were missing responses for between $9-27 \%$ of respondents as follows: Community violence at Wave IV: 9\%; Alcohol or drug use: 15\%; Physical IPV at Wave III: 18\%; Psychological IPV at Wave III: $18 \%$; Sexual IPV at Wave III: $18 \%$; Adolescent community violence: $19 \%$; Adolescent IPV: 24\%; and Child maltreatment: $27 \%$. Therefore, multiple imputation by chained equations (MICE; $m=100$ ) was used to restore the analysis sample to the full, eligible size of 11,500 respondents.

\section{Demographic Characteristics}

Table 1 presents descriptive statistics for the demographic variables for the complete sample. The sample had slightly more females $(n=6,323)$ than males $(n=5,177)$. In the complete sample, most respondents identified as non-Hispanic White $(66.21 \%$ ), and $11.69 \%$ identified as Hispanic, $15.66 \%$ identified as non-Hispanic Black, and $6.44 \%$ identified as another race or ethnicity ("Other"). The average age at Wave III was 21.61 years and at Wave IV was 28.10 years. Nearly one-third of the sample had at least one parent who had

\footnotetext{
${ }^{2}$ Variable name in Add Health: gswgt134.
} 
Table 1 Demographics and Adolescent Risk Behaviors $(n=11,500)$

\begin{tabular}{ll}
\hline & $\%(\mathrm{SE})$ \\
\hline Demographics & \\
Biological sex \% $(n)$ & \\
Male & $49.90(5,177)$ \\
Female & $50.10(6,323)$ \\
Race/Ethnicity & \\
Non-Hispanic White & $66.21(2.75)$ \\
Hispanic & $11.69(1.61)$ \\
Non-Hispanic Black & $15.66(1.99)$ \\
Other & $6.44(0.80)$ \\
Age (Wave III) Mean (SE) & $21.61(0.11)$ \\
Age (Wave IV) Mean (SE) & $28.10(0.11)$ \\
Highest parental education (SES) & \\
Less than high school graduation & $11.65(1.13)$ \\
High school graduate/GED & $27.04(1.16)$ \\
Some college or Vocational education & $29.41(0.90)$ \\
College graduate or beyond & $31.91(1.80)$ \\
Family structure & \\
Two biological parents & $56.82(1.24)$ \\
Two parents (one non-biological) & $16.29(0.51)$ \\
Single mother/Single father & $22.12(0.93)$ \\
Other & $4.77(0.40)$ \\
Adolescent Risk Behaviors & \\
Alcohol or drug use & $11.20(0.51)$ \\
Binge drinking (monthly prior 12 months) & \\
Marijuana use (ever) & $36.16(1.30 .30)$ \\
Cocaine use (ever) & $5.44(0.44)$ \\
Illegal drug (other) or inhalant use (ever) \\
Shoplifting & $15.67(0.75)$ \\
Running away from home & $31.98(0.88)$ \\
\hline
\end{tabular}

$\%$ (percentage) and SE (standard error) are weighted to be population-based estimates and account for multiple imputation

n (sample size) graduated from college or beyond $(31.91 \%)$, while $29.41 \%$ had a parent who had attended some college, $27.04 \%$ had a parent who had graduated high school, and $11.65 \%$ had parents with less than a high school education. Based on family structure during adolescence, most primarily resided with two biological parents (56.82\%). Less common family structures included residing with two parents (one of whom was a non-biological parent; $16.29 \%$ ), with a single mother or single father $(22.12 \%)$, or in some alternative family structure $(4.77 \%)$.

\section{Adolescent Risk Behaviors}

Descriptive statistics for adolescent risk behaviors are presented at the end of Table 1. Alcohol or drug use was the most common risk behavior (43.62\%), followed by shoplifting (31.98\%) and running away from home (11.20\%). Of the substances captured by the alcohol or drug use measure, marijuana was the most common substance used, with $36.16 \%$ of respondents reporting usage during adolescence. Nearly one in five respondents reported monthly binge drinking $(19.14 \%)$, while use of other illegal drugs or inhalants (15.67\%) and cocaine $(5.44 \%)$ was somewhat less common.

\section{Childhood and Adolescent Experiences}

With regards to abuse, violence, or maltreatment in adolescence or childhood as shown in Table 2, $4.21 \%$ of respondents reported exchanging sex as a minor, and this MSE was moderately more common among males $(5.40 \%)$ than females (3.03\%). Approximately one-fourth of respondents experienced community violence $(25.66 \%)$ or IPV (23.63\%), and $39.94 \%$ experienced some form of interpersonal violence victimization in adolescence. Community violence was more common for males (35.74\%) than females (15.62\%), though the proportion of IPV experienced was
Table 2 Childhood and Adolescent Experiences $(n=11,500)$

\begin{tabular}{llll}
\hline & $\begin{array}{l}\text { All } \\
\%(\mathrm{SE})\end{array}$ & $\begin{array}{l}\text { Males } \\
\%(\mathrm{SE})\end{array}$ & $\begin{array}{l}\text { Females } \\
\%(\mathrm{SE})\end{array}$ \\
\hline $\begin{array}{l}\text { Minor Sex Exchange } \\
\text { Ever exchanged sex as a minor }\end{array}$ & $4.21(0.38)$ & $5.40(0.56) *$ & $3.03(0.39)^{*}$ \\
$\begin{array}{l}\text { Adolescent Violence Victimization } \\
\text { Any violence }\end{array}$ & $39.94(1.10)$ & $45.95(1.45) *$ & $33.96(1.13) *$ \\
$\quad \begin{array}{l}\text { Any community violence } \\
\text { Any partner violence }\end{array}$ & $25.66(0.94)$ & $35.74(1.33) *$ & $15.62(0.84)^{*}$ \\
Child Maltreatment & $23.63(0.90)$ & $22.75(1.11)$ & $24.51(1.07)$ \\
$\quad$ Any child maltreatment & $33.77(0.82)$ & $35.53(1.06) *$ & $32.00(1.03) *$ \\
\hline
\end{tabular}

$\%$ (percentage) and SE (standard error) are weighted to be population-based estimates and account for multiple imputation

*significant difference between male vs. female estimates $(p<0.01)$ 
Table 3 Young Adulthood Violence Victimizations $(n=11,500)$

$\%(\mathrm{SE})$

\begin{tabular}{ll} 
& $\%(\mathrm{SE})$ \\
\hline Young Adulthood Violence Victimization & \\
Any violence & $47.47(0.86)$ \\
Any community violence & $14.87(0.67)$ \\
Any partner violence & $41.43(0.79)$ \\
Wave III Violence Outcomes & \\
Any violence & $35.68(0.77)$ \\
Any community violence & $8.15(0.45)$ \\
Any partner violence & $31.66(0.76)$ \\
Physical & $19.22(0.64)$ \\
Psychological & $23.70(0.75)$ \\
Sexual & $12.04(0.46)$ \\
Count of IPV types experienced & \\
0 & $68.34(0.76)$ \\
1 & $13.97(0.51)$ \\
2 & $12.08(0.49)$ \\
3 & $5.60(0.37)$ \\
Wave IV Violence Outcomes & \\
Any violence & $23.57(0.76)$ \\
Any community violence & $8.03(0.43)$ \\
Any partner violence & $18.29(0.66)$ \\
Physical & $9.25(0.54)$ \\
Psychological & $14.43(0.59)$ \\
Sexual & $4.56(0.29)$ \\
Count of IPV types experienced & \\
0 & $81.71(0.66)$ \\
1 & $9.83(0.44)$ \\
3 & $6.98(0.40)$ \\
\hline & $1.49(0.17)$ \\
\hline
\end{tabular}

$\%$ (percentage) and SE (standard error) are weighted to be population-based estimates and account for multiple imputation

Community violence (Someone pulled a knife or gun on them; shot; stabbed; cut; or jumped them (in the past 12 months)) and partner violence (IPV: physical-Partner slapped, hit, or kicked them; or had ever caused an injury, such as a sprain, bruise, or cut (in the past 12 months); psychological-Partner threatened them with violence, pushed or shoved them, or thrown something at them that could hurt (in the past 12 months); sexual-Partner insisted or made them have sexual relations when they did not want to (in the past 12 months)) assessed at Waves III and IV and pooled across waves to capture violence victimization in young adulthood

Any violence represents community violence and/or IPV

Count of IPV types represents a tabulation of the types of IPV reported by a respondent at both Wave III and Wave IV

approximately the same for males $(22.75 \%)$ and females $(24.51 \%)$.

\section{Young Adulthood Violence Victimizations}

Nearly half of the sample (47.47\%) experienced some form of violence in young adulthood (Table 3). IPV was the most prevalent form of violence reported (41.43\%). In Wave III, nearly one-third of respondents reported IPV in at least one relationship (31.66\%). Psychological IPV was the most common form of IPV reported in Wave III (23.70\%), followed by physical (19.22\%) and sexual (12.04\%) IPV. In tabulating the types of IPV reported at Wave III, $13.97 \%$ reported one type of IPV only, $12.08 \%$ reported two types of IPV, and 5.60\% reported experiencing physical, psychological, and sexual IPV in the previous year's relationships. In relation to Wave III, the prevalence of any IPV (18.29\%) was lower in Wave IV, likely due to respondents only reporting on a single relationship, rather than all partnerships from the previous year. Psychological violence remained the most common form of IPV (14.43\%), followed by physical $(9.25 \%)$ and sexual IPV (4.56\%). When considering the types of IPV experienced, 9.83\% experienced one form of IPV, 6.98\% experienced two forms of IPV, and $1.49 \%$ experienced physical, psychological, and sexual IPV in the prior year.

\section{MSE Associated with Young Adulthood Violence Victimizations}

Table 4 presents the bivariate odds ratios for each of the three types of interpersonal violence experienced in young adulthood. Respondents who previously experienced MSE had significant bivariate odds ratios for community violence (OR: 2.08; 95\% CI: 1.47-2.94) and any violence (OR: 1.47; 95\% CI: 1.08-2.01) in young adulthood, though young adulthood IPV was not significant (OR: 1.38; 95\% CI: 1.00-1.90). The remaining adolescent/childhood variables did have a significant bivariate relationship with each of the three violence outcomes. For example, adolescent community violence and IPV were significantly associated with violence victimization for all three young adulthood measures (adolescent community violence-OR for IPV outcome: 1.82; 95\% CI: 1.57-2.10; OR for community violence outcome: 3.07; 95\% CI: 2.60-3.63; OR for any violence outcome: 2.16; 95\% CI: 1.85-2.53; and adolescent IPV—OR for IPV outcome: 2.06; 95\% CI: 1.79-2.37; OR for community violence outcome: 1.45 ; $95 \%$ CI: $1.22-1.73$; OR for any violence outcome: 2.07 ; 95\% CI: 1.80-2.38). Female respondents were less likely to experience community violence (OR: 0.38 ; 95\% CI: 0.33-0.45) and any violence (OR: 0.80 ; $95 \%$ CI: $0.71-0.89$ ) in young adulthood compared to the male respondents. Respondents who were Hispanic or Non-Hispanic Black were more likely to experience all three forms of young adulthood violence, as were those with an adolescent family structure that did not include two biological parents or whose highest parental education was less than a high school degree.

Multivariable logistic regression model results are presented in Table 5 where young adulthood violence victimization outcomes are modeled using childhood and adolescent 
Table 4 Bivariate Odds Ratios and Associated 95\% CIs Estimating Young Adulthood Violence Outcomes (IPV; Community Violence; Any Violence) $(n=11,500)$

\begin{tabular}{|c|c|c|c|}
\hline & $\begin{array}{l}\text { IPV } \\
\text { OR }(95 \% \mathrm{CI})\end{array}$ & $\begin{array}{l}\text { Community Violence } \\
\text { OR }(95 \% \text { CI })\end{array}$ & $\begin{array}{l}\text { Any Violence } \\
\text { OR }(95 \% \text { CI })\end{array}$ \\
\hline Minor sex exchange (MSE) & $1.38(1.00,1.90)$ & $2.08 *(1.47,2.94)$ & $1.47 *(1.08,2.01)$ \\
\hline \multicolumn{4}{|l|}{ Adolescent variables } \\
\hline Community violence & $1.82 *(1.57,2.10)$ & $3.07 *(2.60,3.63)$ & $2.16 *(1.85,2.53)$ \\
\hline Intimate partner violence (IPV) & $2.06 *(1.79,2.37)$ & $1.45 *(1.22,1.73)$ & $2.07 *(1.80,2.38)$ \\
\hline Alcohol or drug use & $1.60 *(1.43,1.79)$ & $1.92 *(1.62,2.28)$ & $1.76 *(1.57,1.97)$ \\
\hline Shoplifting & $1.54 *(1.38,1.72)$ & $1.71 *(1.46,1.99)$ & $1.70 *(1.51,1.92)$ \\
\hline Running away from home & $1.71 *(1.44,2.03)$ & $1.86 *(1.48,2.33)$ & $1.87 *(1.58,2.19)$ \\
\hline \multicolumn{4}{|l|}{ Childhood variable } \\
\hline Maltreatment & $1.70 *(1.51,1.91)$ & $1.74 *(1.47,2.06)$ & $1.75 *(1.57,1.96)$ \\
\hline \multicolumn{4}{|l|}{ Demographics } \\
\hline \multicolumn{4}{|l|}{ Biological sex } \\
\hline Male & 1.00 (ref) & 1.00 (ref) & 1.00 (ref) \\
\hline Female & $0.97(0.87,1.09)$ & $0.38 *(0.33,0.45)$ & $0.80 *(0.71,0.89)$ \\
\hline \multicolumn{4}{|l|}{ Race/Ethnicity } \\
\hline Non-Hispanic White & 1.00 (ref) & 1.00 (ref) & 1.00 (ref) \\
\hline Hispanic & $1.33 *(1.12,1.58)$ & $1.53 *(1.21,1.92)$ & $1.44 *(0.21,1.72)$ \\
\hline Non-Hispanic Black & $1.57 *(1.33,1.86)$ & $1.98 *(1.54,2.56)$ & $1.74 *(1.46,2.08)$ \\
\hline Other & $1.13(0.89,1.42)$ & $1.09(0.78,1.52)$ & $1.15(0.91,1.44)$ \\
\hline Age (Wave IV) & $0.98(0.95,1.02)$ & $0.91 *(0.86,0.96)$ & $0.96 *(0.92,1.00)$ \\
\hline \multicolumn{4}{|l|}{ Highest parental education (SES) } \\
\hline Less than high school graduation & $1.34 *(1.13,1.59)$ & $1.69 *(1.37,2.09)$ & $1.48 *(1.24,1.78)$ \\
\hline High school graduate/GED or beyond & 1.00 (ref) & 1.00 (ref) & 1.00 (ref) \\
\hline \multicolumn{4}{|l|}{ Family structure } \\
\hline Two biological parents & 1.00 (ref) & 1.00 (ref) & 1.00 (ref) \\
\hline Two parents (one non-biological) & $1.23 *(1.07,1.42)$ & $1.62 *(1.30,2.01)$ & $1.28 *(1.11,1.48)$ \\
\hline Single mother/Single father & $1.33 *(1.15,1.53)$ & $1.97 *(1.64,2.37)$ & $1.48 *(1.28,1.71)$ \\
\hline Other & $1.30 *(1.01,1.69)$ & $2.34 *(1.66,3.29)$ & $1.51 *(1.18,1.93)$ \\
\hline
\end{tabular}

Odds Ratio (OR), 95\% Confidence Interval (CI)

*significant OR

(ref): reference category variables and demographic controls. For each of these three models and for the bivariate results presented in Table 4, preliminary examinations investigated the interaction effect between biological sex and the independent variables of each model. Biological sex did not significantly $(\alpha=0.05)$ impact any of the associations between any of the independent variables and the outcomes of experiencing violence, so no stratified results for males and females are presented in the bivariate or multivariable models.

The IPV model of Table 5 presents the results of the logistic regression model examining how MSE is associated with any IPV experiences in young adulthood, while controlling for other interpersonal violence in adolescence, adolescent risk behaviors, child maltreatment, and demographic characteristics. MSE was not significantly associated with young adulthood IPV (aOR: 1.05; 95\% CI: 0.74-1.48). Beyond MSE, all other adolescent variables were significant: alcohol or drug use (aOR: 1.28, 95\% CI: 1.11-1.46), shoplifting (aOR: 1.16, 95\% CI: 1.02-1.32), running away from home (aOR: $1.21,95 \%$ CI: 1.00-1.45), community violence (aOR: 1.40, 95\% CI: 1.18-1.64), and IPV (aOR: 1.77, 95\% CI: 1.52-2.06). Respondents who experienced child maltreatment were also more likely to experience IPV in young adulthood (aOR: 1.52, 95\% CI: 1.34-1.71). NonHispanic Black respondents had significantly increased odds of experiencing IPV (aOR: 1.49, 95\% CI: $1.27-1.76$ ), as did respondents whose parents had less than a high school education (aOR: 1.25, 95\% CI: 1.04-1.50). Age had a moderately protective effect with each additional year of life modestly lowering odds of experiencing IPV in the previous year (aOR: 0.93, 95\% CI: 0.90-0.97).

The community violence model of Table 5 presents the results of the multivariable regression model of community violence in young adulthood as a function of MSE and adolescent, childhood, and demographic control variables. Although community violence during adulthood had 
Table 5 Adjusted Odds Ratios and Associated 95\% CIs Estimating Young Adulthood Violence Outcomes as a Function of MSE, Adolescent and Childhood Variables, and Demographic Variables $(n=11,500)$

\begin{tabular}{|c|c|c|c|}
\hline & $\begin{array}{l}\text { IPV } \\
\text { aOR }(95 \% \mathrm{CI})\end{array}$ & $\begin{array}{l}\text { Community Violence } \\
\text { aOR }(95 \% \mathrm{CI})\end{array}$ & $\begin{array}{l}\text { Any Violence } \\
\text { aOR }(95 \% \text { CI) }\end{array}$ \\
\hline Minor sex exchange (MSE) & $1.06(0.75,1.50)$ & $1.37(0.93,2.03)$ & $1.06(0.76,1.50)$ \\
\hline \multicolumn{4}{|l|}{ Adolescent variables } \\
\hline Community violence & $1.40 *(1.19,1.65)$ & $1.90 *(1.57,2.32)$ & $1.53 *(1.28,1.83)$ \\
\hline Intimate partner violence (IPV) & $1.77 *(1.52,2.07)$ & $1.15(0.94,1.40)$ & $1.75 *(1.50,2.04)$ \\
\hline Alcohol or drug use & $1.28 *(1.11,1.46)$ & $1.64 *(1.35,1.99)$ & $1.40 *(1.22,1.60)$ \\
\hline Shoplifting & $1.16 *(1.02,1.32)$ & $1.06(0.88,1.28)$ & $1.21 *(1.06,1.38)$ \\
\hline Running away from home & $1.21 *(1.01,1.46)$ & $1.32 *(1.02,1.72)$ & $1.28 *(1.07,1.53)$ \\
\hline \multicolumn{4}{|l|}{ Childhood variable } \\
\hline Maltreatment & $1.52 *(1.34,1.71)$ & $1.44 *(1.20,1.72)$ & $1.53 *(1.36,1.72)$ \\
\hline \multicolumn{4}{|l|}{ Demographics } \\
\hline \multicolumn{4}{|l|}{ Biological sex } \\
\hline Male & 1.00 (ref) & 1.00 (ref) & 1.00 (ref) \\
\hline Female & $1.05(0.93,1.19)$ & $0.42 *(0.35,0.50)$ & $0.86 *(0.76,0.98)$ \\
\hline \multicolumn{4}{|l|}{ Race/Ethnicity } \\
\hline Non-Hispanic White & 1.00 (ref) & 1.00 (ref) & 1.00 (ref) \\
\hline Hispanic & $1.13(0.94,1.36)$ & $1.16(0.89,1.50)$ & $1.18(0.98,1.43)$ \\
\hline Non-Hispanic Black & $1.50 *(1.27,1.78)$ & $1.71 *(1.33,2.18)$ & $1.64 *(1.39,1.95)$ \\
\hline Other & $1.03(0.80,1.32)$ & $0.95(0.68,1.33)$ & $1.03(0.81,1.32)$ \\
\hline Age (Wave IV) & $0.93 *(0.90,0.97)$ & $0.82 *(0.78,0.87)$ & $0.90 *(0.86,0.94)$ \\
\hline \multicolumn{4}{|l|}{ Highest parental education (SES) } \\
\hline Less than high school graduation & $1.25 *(1.04,1.50)$ & $1.42 *(1.11,1.82)$ & $1.34 *(1.10,1.62)$ \\
\hline High school graduate/GED or beyond & 1.00 (ref) & 1.00 (ref) & 1.00 (ref) \\
\hline \multicolumn{4}{|l|}{ Family structure } \\
\hline Two biological parents & 1.00 (ref) & 1.00 (ref) & 1.00 (ref) \\
\hline Two parents (one non-biological) & $1.06(0.92,1.23)$ & $1.39 *(1.12,1.72)$ & $1.09(0.94,1.26)$ \\
\hline Single mother/Single father & $1.02(0.88,1.18)$ & $1.44 *(1.18,1.76)$ & $1.10(0.94,1.28)$ \\
\hline Other & $0.93(0.70,1.23)$ & $1.83 *(1.23,2.74)$ & $1.06(0.80,1.41)$ \\
\hline
\end{tabular}

Adjusted Odds Ratio (aOR), 95\% Confidence Interval (CI)

*significant aOR

(ref): reference category a significant bivariate relationship with MSE, MSE was not a significantly associated with community violence in young adulthood in the multivariable model (aOR: 1.37, 95\% CI: 0.93-2.03). Of the other adolescent variables, alcohol or drug use (aOR: 1.64, 95\% CI: 1.35-1.99), running away from home (aOR: $1.33,95 \%$ CI: $1.02-1.73)$, and community violence (aOR: $1.90,95 \%$ CI: $1.56-2.31$ ) were significantly associated with community violence in young adulthood. Shoplifting (aOR: 1.06, 95\% CI: 0.88-1.28) and adolescent IPV (aOR: 1.15, 95\% CI: 0.94-1.40) were not significantly associated with the community violence outcome. Child maltreatment was significantly associated with community violence in young adulthood (aOR: 1.43, 95\% CI: 1.20-1.71). Non-Hispanic Black respondents had significantly increased odds of experiencing community violence (aOR: 1.71, 95\% CI: 1.34-2.19), as well as respondents whose parents had less than a high school education (aOR: $1.42,95 \%$ CI: $1.11-1.82)$. Respondents who were in any family structure in adolescence other than two biological parents also had significantly increased odds of experiencing community violence (Two parents (at least one non-biological): aOR: $1.39,95 \%$ CI: 1.12-1.72; Single mother/Single father: aOR: $1.44,95 \%$ CI: 1.18-1.76; Other: aOR: 1.83 , 95\% CI: 1.23-2.74). Protective associations against community violence were found for females (aOR: $0.42,95 \% \mathrm{CI}$ : $0.35-0.50)$ and for each additional year of life (aOR: 0.82 , 95\% CI: 0.78-0.87).

The any violence model of Table 5 examines the relationship of MSE and any interpersonal violence in young adulthood, controlling for the other variables. MSE was not significantly associated with interpersonal violence in young adulthood (aOR: 1.05, 95\% CI: 0.75-1.48). All other childhood and adolescent variables had significantly increased odds of interpersonal violence including community violence (aOR: 1.52, 95\% CI: 1.27-1.82), IPV (aOR: 1.75, 95\% CI: $1.50-2.04$ ), alcohol or drug use (aOR: $1.40,95 \%$ CI: 
1.22-1.60), shoplifting (aOR: 1.21 , 95\% CI: 1.06-1.39), running away from home (aOR: $1.27,95 \% \mathrm{CI}: 1.07-1.52)$, child maltreatment (aOR: 1.52, 95\% CI: 1.35-1.72). NonHispanic Black respondents also had significantly increased odds of any young adulthood violence (aOR: 1.64, 95\% CI: 1.38-1.94), as did respondents whose parents had not completed high school (aOR: 1.34, 95\% CI: 1.10-1.62). Each additional year of life moderately protected a respondent from violence (aOR: 0.90, 95\% CI: 0.86-0.94) and females were also modestly protected from the violence outcome compared to males (aOR: 0.86, 95\% CI: 0.76-0.98).

\section{Discussion}

This study investigated potential associations between MSE and violence outcomes in young adulthood. Bivariate regressions supported Hypothesis 1 and indicated that community violence, but not IPV, victimizations in young adulthood were more likely for those with a history of MSE. However, after controlling for childhood, adolescent, and demographic variables in the multivariable models, these connections between MSE and forms of young adulthood violence (Hypothesis 1) were not statistically significant. In the multivariable models which control for adolescent and childhood variables along with demographic variables, individuals who exchanged sex for money or drugs as minors did not have a distinctly higher or lower likelihood of young adulthood violence victimization than their peers who did not exchange sex as minors. Preliminary investigations in the bivariate and multivariable logistic regression models found that the reported odds ratios did not significantly differ between males and females. Thus, the significant connections for child maltreatment, adolescent risk behaviors, and adolescent IPV and community violence to young adulthood violence, along with the non-significant associations between MSE and young adulthood violence, were not significantly different for males and females.

This study did find significant connections between abuse or violence in childhood and adolescence and violence victimizations experienced in young adulthood (Hypothesis 2). Adolescent experiences of IPV and community violence were each associated with increased likelihood of experiencing IPV in young adulthood. Adolescent community violence experiences also were significantly linked to the likelihood of community violence victimizations in young adulthood. For both of these young adult violence outcomes, child maltreatment and adolescent risk behaviors of alcohol or drug use, shoplifting, and running away from home were significantly associated with later violence victimization in adulthood.

These findings build and expand on previous research, particularly with regards to the biological sex of individuals experiencing MSE (a form of DMST), the links between forms of violence across the life course, and the connections between adolescent behaviors and violence victimizations. Unlike the frequently-stated assumption in the antitrafficking field that most minors involved in commercial sex are females, in the population-based Add Health sample the majority of respondents reporting MSE were male $(63.98 \%)$ rather than female $(36.02 \%)$, which has been noted and discussed in previous publications (Edwards et al., 2006; Kaestle, 2012; Ulloa et al., 2016). Though present findings did not demonstrate a significant link between MSE and subsequent interpersonal violence in young adulthood in the multivariable regression models, the results connecting maltreatment and violence in childhood and adolescence to young adulthood violence victimizations are in-line with findings from previous studies. Consistent with these models, child abuse has been shown to increase the odds of adulthood community violence and IPV victimization, and IPV victimization in adolescence has been linked to subsequent young adulthood IPV victimizations (Desai et al., 2002; Gömez, 2011; Iratzoqui \& Watts, 2016; Smith et al., 2003). The multivariable results build on previous investigations using Add Health data wherein community violence was significantly predictive of adulthood IPV victimization for males but not for females (Fang \& Corso, 2007). The link between adolescent community violence victimization and community violence victimization in young adulthood is consistent with findings showing that persistent violence victimizations across adolescence increases the risk for community violence victimization in Wave IV of Add Health data, which represents the young adult time period (ages: 24-34 years) (Tillyer, 2014). The significant associations between risky adolescent behaviors and adulthood violence may support the risky lifestyles model in which involvement in risky behaviors - including drug and alcohol useincreases the likelihood of violence victimization, though there is a need for better theory to explain these longitudinal links (Iratzoqui \& Watts, 2016).

These findings highlight the relevance of the life course perspective when considering violence experienced by young adults, as these violent events are not isolated but clearly linked to child abuse/maltreatment and adolescent interpersonal violence. These repeat victimizations across the life course may not only augment an individual's risk for additional victimization but may meaningfully impact violence survivors by affecting how they respond to or adapt to each additional experience of abuse or violence (Iratzoqui \& Watts, 2016). Victimization across the life course has been referred to as a "victim careers pathway" in previous work, and understanding these repeat victimizations may be essential to improve violence prevention and intervention efforts (Tillyer, 2014). As noted in the work by Herrenkohl et al. (2020) these life course patterns of violence 
victimizations may be explained by psychobiological processes, which connect exposure to adversity via adverse events (e.g., child maltreatment) to changes in patterns of arousal, stress response, and stress regulation. Shifts in these patterns can cause children and adolescents to engage in risky and/or impulsive behaviors such as violence, substance use, or risky sexual behaviors (Herrenkohl et al., 2020). Further exposure to violence in adolescence can exacerbate an individual's maladapted stress response and increase the risks for additional victimizations in adulthood (Herrenkohl et al., 2020). These processes - though potentially influenced by other complex physiological and psychological responsesexplain how childhood and adolescent victimization experiences can continue across the life course and into adulthood via revictimization and/or polyvictimization events. Though MSE was significantly associated with young adulthood community violence in the bivariate regression model but not significantly linked to violence in young adulthood in these multivariable analyses, sex trafficking of minors has been found to be linked with community violence in adolescence (Franchino-Olsen et al., 2021). The null results for MSE in the fully-adjusted models may be due to imprecision of the MSE measure-in which we cannot discern the context of the MSE event or how the experience was conceptualized by the minor (e.g., whether viewed as upsetting or abusive), both of which may be critical to the outcomes stemming from the event—or may accurately reflect the lack of long-term violence outcomes for individuals with a history of MSE. Given the adolescent links between community violence and DMST/MSE, it is possible that the violence associations presented here and found previously for violence in adolescence represent an interconnected web of violence of poly-victimization in adolescence and adulthood and repeat victimization from childhood into adulthood.

The implications of these violence connections are relevant for violence prevention, intervention, and screening efforts. Targeted prevention efforts for violence in adolescence or young adulthood should consider how previous experience of violence victimization impacts these populations and their needs. Violence intervention or screening efforts should be informed by the likelihood that the focus of the intervention or screening may not be an isolated victimization experience in a survivor's life and may not represent the most upsetting or concerning form of violence they have experienced (Twis, 2020). By viewing any experiences of violence, including MSE events, through the life course lens, violence prevention and response efforts can operate with sensitivity for prior adverse events and victimizations and seek to address the interconnected events while aiming to prevent future violent events. Such anti-violence efforts must be survivor-centered and trauma-informed in order to adequately respond to the complex needs and to prioritize the agency of survivors of repeat victimizations (U.S.
Department of Health and Human Services Administration for Children Youth and Families (ACYF), 2013). All policy seeking to address MSE, IPV, or community violence in adolescence or young adulthood must take a holistic approach and consider the interconnected nature of violence rather than seeking for siloed responses to single forms of violence.

\section{Limitations}

Characteristics of Add Health contribute to the limitations of this study. Wave I and Wave II surveys were collected from 1994 to 1996, which raises whether contexts and correlates for the MSE measure have been substantially altered in the past 25 years. Despite the age of the data, minors may continue to be sufficiently unprotected from abuse and exploitation as in the mid-1990s, especially because many of the factors that are linked to or create trafficking vulnerability (e.g., racism, poverty, child abuse and maltreatment) remain social concerns. Findings from studies using Add Health data to investigate risk and protective factors for and the outcomes stemming from MSE are similar to results from DMST samples collected more recently, which seems encouraging and may indicate the continued relevance of the Add Health measures to the DMST field (Edwards et al., 2006; Fedina et al., 2019; Franchino-Olsen, 2019; Gerassi et al., 2021; Havlicek et al., 2016; Kaestle, 2012; Le et al., 2018; Ulloa et al., 2016). However, the age of the data should not be overlooked as a limitation.

Though intended to capture non-partner violence in Add Health, the questions used to assess community violence (assault, threatened or harmed with a weapon) may reflect some incidents of violence occurring within an intimate partnership and be better designated as IPV. The phrasing of the questions asked respondents for the MSE measures (Have you ever given someone sex in exchange for drugs or money?) introduces the possibility that some respondents answered affirmatively while actually meaning they acted as buyers in a sex exchange. It is unclear how many respondents answered in this unintended way compared to how many did not answer affirmatively due to stigma or shame despite a history of MSE experiences. Despite the diversity added to this study via the inclusion of males who experienced MSE, the Add Health sample is lacking in measures of gender diversity by not being able to assess gender identity at Wave I or Wave II. As the only available measure is male or female biological sex, we were not able to assess how these relationships vary for respondents who are transgender, gender non-conforming, or gender non-binary. This is particularly relevant when considering minor sex trafficking, as previous work has noted that minors who identify as a gender minority may experience a disproportionate burden of DMST (Choi, 2015; Fedina et al., 2019; IOM \& NRC, 2013). The MSE 
events captured via the Add Health measure are lacking in detail about these events as it does not allow the distinction of the context of the MSE events (e.g., coerced versus needs-based exchanges). It also does not provide any data on how the minors conceptualized these events (e.g., Did adolescents view their MSE as entirely voluntary or find the experience traumatic?). The lack of granularity around this measure may explain the non-significant associations between MSE and young adulthood violence. Future research may be required to explore the circumstances around and survivors' conceptualizations of the DMST or MSE events to determine whether those details impact risk of violence beyond adolescence.

\section{Conclusion}

This study did not find that those who experienced MSE were more likely than their peers to experience violence victimization during young adulthood violence after controlling for other forms of violence and additional adolescent and demographic factors. However, findings did support the links between child maltreatment and young adulthood interpersonal violence and between adolescent interpersonal violence (community violence, IPV) and young adulthood interpersonal violence. By demonstrating the connected nature of violence and abuse across the life course, these findings highlight how prevention, intervention, or response efforts targeted at adolescent violence may have far-reaching effects by decreasing the risk of interpersonal violence in young adulthood. The link between child maltreatment and young adulthood violence also reinforces how upstream the risk for adulthood violence begins, spotlighting the need to protect vulnerable children and adolescents from facing any form of abuse, maltreatment, or violence.

Supplementary Information The online version contains supplementary material available at https://doi.org/10.1007/s10896-021-00352-8.

Acknowledgements This research uses data from Add Health, a program project directed by Kathleen Mullan Harris and designed by J. Richard Udry, Peter S. Bearman, and Kathleen Mullan Harris at the University of North Carolina at Chapel Hill, and funded by grant P01-HD31921 from the Eunice Kennedy Shriver National Institute of Child Health and Human Development, with cooperative funding from 23 other federal agencies and foundations. Information on how to obtain the Add Health data files is available on the Add Health website (https://addhealth.cpc.unc.edu). No direct support was received from grant P01-HD31921 for this analysis.

Note: Use of this acknowledgment requires no further permission from the persons named.

Funding Research reported in this publication was supported by NICHD of the National Institutes of Health under award number P2C HD050924.

\section{Declarations}

Competing interest No potential competing interests were reported by the authors.

Open Access This article is licensed under a Creative Commons Attribution 4.0 International License, which permits use, sharing, adaptation, distribution and reproduction in any medium or format, as long as you give appropriate credit to the original author(s) and the source, provide a link to the Creative Commons licence, and indicate if changes were made. The images or other third party material in this article are included in the article's Creative Commons licence, unless indicated otherwise in a credit line to the material. If material is not included in the article's Creative Commons licence and your intended use is not permitted by statutory regulation or exceeds the permitted use, you will need to obtain permission directly from the copyright holder. To view a copy of this licence, visit http://creativecommons.org/licenses/by/4.0/.

\section{References}

Choi, K. R. (2015). Risk factors for domestic minor sex trafficking in the United States. Journal of Forensic Nursing, 11(2), 66-76. https://doi.org/10.1097/JFN.0000000000000072

Classen, C. C., Palesh, O. G., \& Aggarwal, R. (2005). Sexual revictimization: A review of the empirical literature. Trauma, Violence, \& Abuse, 6(2), 103-129. https://doi.org/10.1177/1524838005275087

Costa, B. M., Kaestle, C. E., Walker, A., Curtis, A., Day, A., Toumbourou, J. W., \& Miller, P. (2015). Longitudinal predictors of domestic violence perpetration and victimization: A systematic review. Agression and Violent Behavior, 24, 261-272. https://doi. org/10.1016/j.avb.2015.06.001

Cui, M., Ueno, K., Gordon, M., \& Fincham, F. D. (2013). The continuation of intimate partner violence from adolescence to young adulthood. Journal of Marriage and Family, 75(2), 300-313. https://doi.org/10.1111/jomf.12016

Decker, M. R., Wilcox, H. C., Holliday, C. N., \& Webster, D. W. (2018). An integrated public health approach to interpersonal violence and suicide prevention and response. Public Health Reports, 133(Supplement 1), 65S-79S. https://doi.org/10.1177/ 0033354918800019

Desai, S., Arias, I., Thompson, M. P., \& Basile, K. C. (2002). Childhood victimization and subsequent adult revictimization assessed in a nationally representative sample of women and men. Violence and Victims, 17(6), 639-653. https://doi.org/10.1891/vivi. 17.6.639.33725

Edinburgh, L., Pape-Blabolil, J., Harpin, S. B., \& Saewyc, E. (2015). Assessing exploitation experiences of girls and boys seen at a Child Advocacy Center. Child Abuse \& Neglect, 46, 47-59. https://doi.org/10.1016/j.chiabu.2015.04.016

Edwards, J. M., Iritani, B. J., \& Hallfors, D. D. (2006). Prevalence and correlates of exchanging sex for drugs or money among adolescents in the United States. Sexually Transmitted Infections, 82(5), 354-358. https://doi.org/10.1136/sti.2006.020693

Elder, G. H. (1985). Perspectives on the life course. Life course dynamics: Trajectories and transitions (pp. 23-49). Cornell University Press.

Exner-Cortens, D., Eckenrode, J., Bunge, J., \& Rothman, E. (2017). Revictimization after adolescent dating violence in a matched, national sample of youth. Journal of Adolescent Health, 60(2), 176-183. https://doi.org/10.1016/j.jadohealth.2016.09.015 
Fang, X., \& Corso, P. S. (2007). Child maltreatment, youth violence, and intimate partner violence. Developmental relationships. American Journal of Preventive Medicine, 33(4), 281-290. https://doi.org/10.1016/j.amepre.2007.06.003

Fargo, J. D. (2009). Direct and indirect behavioral risk factors across the lifespan. Journal of Interpersonal Violence, 24(11), 1771-1791.

Fedina, L., Williamson, C., \& Perdue, T. (2019). Risk factors for domestic child sex trafficking in the United States. Journal of Interpersonal Violence, 34(13), 2653-2673. https://doi.org/10. $1177 / 0886260516662306$

Franchino-Olsen, H. (2019). Vulnerabilities relevant for commercial sexual exploitation of children/domestic minor sex trafficking: A systematic review of risk factors. Trauma, Violence, and Abuse. https://doi.org/10.1177/1524838018821956

Franchino-Olsen, H., Chesworth, B. R., Boyle, C., Rizo, C. F., Martin, S. L., Jordan, B., ... Stevens, L. (2020). The prevalence of sex trafficking of children and adolescents in the United States: A scoping review. Trauma, Violence, and Abuse. https://doi.org/10. $1177 / 1524838020933873$

Franchino-Olsen, H., Martin, S. L., Halpern, C. T., Preisser, J. S., Zimmer, C., \& Shanahan, M. (2021). Adolescent experiences of violence victimizations among minors who exchange sex/experience minor sex trafficking. Journal of Interpersonal Violence. https:// doi.org/10.1177/08862605211021967

Gerassi, L. B., Cheng, S. Y., Muentner, L., \& Benson, M. (2021). Prevalence and associated characteristics of youth who trade sex in a representative sample of high school students. Journal of Adolescence, 93, 1-9. https://doi.org/10.1016/j.adolescence.2021.09.008

Goldberg, A., \& Moore, J. (2018). Domestic minor sex trafficking. Child and Adolescent Psychiatric Clinics of North America, 27(1), 77-92. https://doi.org/10.1016/j.chc.2017.08.008

Gömez, A. M. (2011). Testing the cycle of violence hypothesis: Child abuse and adolescent dating violence as predictors of intimate partner violence in young adulthood. Youth and Society, 43(1), 171-192. https://doi.org/10.1177/0044118X09358313

Harris, K. M. (2013). The Add Health study: Design and accomplishments. Retrieved from http://www.cpc.unc.edu/projects/addhe alth/documentation/guides/DesignPaperWIIV.pdf. Accessed 29 Oct 2020

Harris, K. M., Halpern, C. T., Whitsel, E., Hussey, J., Tabor, J., Entzel, P., Udry, J. R. (2009). The National Longitudinal Study of Adolescent to Adult Health: Research design. Retrieved July 2, 2019, from https://www.cpc.unc.edu/projects/addhealth/design

Havlicek, J., Huston, S., Boughton, S., \& Zhang, S. (2016). Human trafficking of children in Illinois: Prevalence and characteristics. Children and Youth Services Review, 69, 127-135. https://doi.org/ 10.1016/j.childyouth.2016.08.010

Hendricks, J. (2012). Considering life course concepts. Journals of Gerontology - Series B Psychological Sciences and Social Sciences, 67B(2), 226-231. https://doi.org/10.1093/geronb/gbr147

Herrenkohl, T. I., Fedina, L., Roberto, K. A., Raquet, K. L., Hu, R. X., Rousson, A. N., \& Mason, W. A. (2020). Child maltreatment, youth violence, intimate partner violence, and elder mistreatment: A review and theoretical analysis of research on violence across the life course. Trauma, Violence, and Abuse. https://doi.org/10. $1177 / 1524838020939119$

Hing, N., O'mullan, C., Mainey, L., Nuske, E., Breen, H., Taylor, A. (2021). Impacts of male intimate partner violence on women: A life course perspective. International Journal of Environmental Research and Public Health, 18(16). https://doi.org/10.3390/ijerp h18168303

IOM, NRC. (2013). Confronting commercial sexual exploitation and sex trafficking of minors in the United States. https://doi.org/10. $17226 / 18358$
Iratzoqui, A., Watts, S. J. (2016). Longitudinal risks for domestic violence. Journal of Interpersonal Violence, 088626051666389. https://doi.org/10.1177/0886260516663897

Jouriles, E. N., Choi, H. J., Rancher, C., \& Temple, J. R. (2017). Teen dating violence victimization, trauma symptoms, and revictimization in early adulthood. Journal of Adolescent Health, 61(1), 115-119. https://doi.org/10.1016/j.jadohealth.2017.01.020

Kaestle, C. E. (2012). Selling and buying sex: A longitudinal study of risk and protective factors in adolescence. Prevention Science, 13(3), 314-322. https://doi.org/10.1007/s11121-011-0268-8

Kotrla, K. (2010). Domestic minor sex trafficking in the United States. Social Work, 55(2), 181-187. https://doi.org/10.1093/sw/55.2.181

Le, P. D., Ryan, N., Rosenstock, Y., Goldmann, E., Le, P. D., Ryan, N., ... Goldmann, E. (2018). Health issues associated with commercial sexual exploitation and sex trafficking of children in the United States: A systematic review. Behavioral Medicine, 44(3), 219-233. https://doi.org/10.1080/08964289.2018.1432554

National Academies of Sciences Engineering and Medicine. (2020). Estimating the prevalence of human trafficking in the United States: Considerations and complexities. In Estimating the Prevalence of Human Trafficking in the United States: Considerations and Complexitiess: Proceedings of a Workshop. https://doi.org/ $10.17226 / 25614$

O'Brien, J. E. (2017). "What does it matter how we define it?": Exploring definitions of DMST among service providers and victims/ survivors. Journal of Human Trafficking, 00(00), 1-18. https:// doi.org/10.1080/23322705.2017.1410415

Reid, J. A., \& Piquero, A. R. (2014). On the relationships between commercial sexual exploitation/prostitution, substance dependency, and delinquency in youthful offenders. Child Maltreatment, 19(3-4), 247-260. https://doi.org/10.1177/1077559514539752

Robert, N., \& Willis, B. (2013). And boys too. Retrieved from https:// www.ecpatusa.org/ecpat-reports/. Accessed 19 Sept 2019

Showden, C. R., \& Majic, S. (2018). Youth who trade sex in the U.S.: Intersectionality, Agency, and Vulnerability. Temple University Press.

Smith, P. H., White, J. W., \& Holland, L. J. (2003). A longitudinal perspective on dating violence among adolescent and college-age women. American Journal of Public Health, 93(7), 1104-1109. https://doi.org/10.2105/AJPH.93.7.1104

StataCorp. (2020). Stata statistical software: Release 16.1. StataCorp LP

Stransky, M., Finkelhor, D. (2008). How many juveniles are involved in prostitution in the U.S.? (Vol. 03824). Retrieved from http://www. unh.edu/ccrc/prostitution/Juvenile_Prostitution_factsheet.pdf

Tillyer, M. S. (2014). Violent victimization across the life course: Moving a "victim careers" agenda forward. Criminal Justice and Behavior, 41(5), 593-612. https://doi.org/10.1177/0093854813 509370

Trafficking Victims Protection Act. (2017). Pub. L. No. 115-393

Trafficking Victims Protection Reauthorization Act. (2003). Pub. L. No. $108-193$.

Trafficking Victims Protection Reauthorization Act. (2006). Pub. L. No. 109-164.

Trafficking Victims Protection Reauthorization Act. (2013). Pub. L. No. 113-4.

Twis, M. K. (2020). Risk factor patterns in domestic minor sex trafficking relationships. Journal of Human Trafficking, 6(3), 309-326. https://doi.org/10.1080/23322705.2019.1627775

U.S. Department of Health and Human Services Administration for Children Youth and Families (ACYF). (2013). Guidance to states and services on addressing human trafficking of children and youth in the United States. Retrieved from https://www.acf.hhs. gov/cb/resource/human-trafficking-guidance. Accessed 17 Jan 2020 
Ulloa, E., Salazar, M., \& Monjaras, L. (2016). Prevalence and correlates of sex exchange among a nationally representative sample of adolescents and young adults. Journal of Child Sexual Abuse: Research, Treatment, \& Program Innovations for Victims, Survivors, \& Offenders, 25(5), 524-537. https://doi.org/10.1080/10538 712.2016.1167802

Victims of Trafficking and Violence Protection Act. (20000). Pub. L. No. 106-386.
Walls, N. E., \& Bell, S. (2011). Correlates of engaging in survival sex among homeless youth and young adults. The Journal of Sex, 48(5), 423-436. https://doi.org/10.1080/00224499.2010.501916

William Wilberforce Trafficking Victims Protection Reauthorization Act. (2008). Pub. L. No. 110-457.

Publisher's Note Springer Nature remains neutral with regard to jurisdictional claims in published maps and institutional affiliations. 